\title{
Characterization of Human Influenza Viruses in Lebanon during 2010-2011 and 2011-2012 Post-Pandemic Seasons
}

\author{
Hassan Zaraket ${ }^{a, c}$ Clyde Dapat ${ }^{d}$ Soha Ghanem ${ }^{b}$ Zainab Ali ${ }^{b}$ Mireille Lteif ${ }^{b}$ \\ Hiroki Kondo $^{d}$ Isolde C. Dapat ${ }^{d}$ Kousuke Saito ${ }^{d}$ Ghazi Kayalic \\ Hiroshi Suzuki $^{d, e}$ Ghassan Dbaibo $^{\text {b }}$ Reiko Saito $^{d}$ \\ ${ }^{a}$ Department of Experimental Pathology, Immunology and Microbiology, and ${ }^{\mathrm{b}}$ Division of Pediatric Infectious \\ Diseases, Department of Pediatrics and Adolescent Medicine and the Center for Infectious Diseases Research, \\ American University of Beirut, Beirut, Lebanon; ${ }^{C}$ Division of Virology, Department of Infectious Diseases, St. Jude \\ Children's Research Hospital, Memphis, Tenn., USA; ${ }^{\mathrm{d}}$ Division of International Health, Graduate School of Medical \\ and Dental Sciences, Niigata University, and ' School of Nursing, Niigata Seiryo University, Niigata, Japan
}

\section{Key Words}

Influenza virus · Pandemic H1N1 - Neuraminidase inhibitors · Antiviral drug susceptibility · Lebanon · Middle East

\begin{abstract}
Objective: To genetically characterize human influenza viruses and their susceptibilities to antivirals during two post-pandemic seasons in Lebanon. Methods: Influenza virus was isolated from nasopharyngeal swabs that were obtained from patients with influenza-like illness during 2010-2012 and further analyzed both phenotypically and genotypically. Results: During the 2010-2011 season, both 2009 pandemic H1N1 (H1N1p) and B viruses co-circulated with equal prevalence, while the H3N2 virus predominated during the 2011-2012 season. All H3N2 and $\mathrm{H} 1 \mathrm{~N} 1$ viruses were resistant to amantadine. Importantly, all viruses of the influenza $A$ and $B$ types were susceptible to the neuraminidase (NA) inhibitors oseltamivir, zanamivir, peramivir, and laninamivir. Nonetheless, all 2011-2012 H1N1p isolates had three mutations (V2411, N369K, and N386S) in the NA gene that were suggested to be permissive of the $\mathrm{H} 275 \mathrm{Y}$ mutation, which confers resistance to
\end{abstract}

oseltamivir. We also detected one H1N1p virus during the 2010-2011 season with a 4-fold decrease in susceptibility to oseltamivir due to an NA-S247N mutation. This isolate was phylogenetically distinct from other $\mathrm{H} 1 \mathrm{~N} 1 \mathrm{p}$ viruses that were isolated in other regions. Conclusions: Influenza A viruses with reduced susceptibility to oseltamivir and mutations permissive for acquiring NA resistance-conferring mutation with minimal burden on their fitness were isolated in Lebanon.

(c) 2014 S. Karger AG, Basel

\section{Introduction}

Annual influenza outbreaks affect on average 5-20\% of the world's population, resulting in a significant burden on human health [1]. These outbreaks are caused by seasonal influenza viruses that undergo continuous antigenic changes [2]. Occasionally, pandemics can occur due to antigenic shifts in the HA protein. Until the 20082009 winter season, two influenza A subtypes, H3N2 and $\mathrm{H} 1 \mathrm{~N} 1$, and influenza B circulated in humans. However, in spring 2009, a novel influenza virus (pandemic H1N1 2009; H1N1p) emerged and quickly spread around the

\section{KARGER}

E-Mail karger@karger.com

www.karger.com/int
(C) 2014 S. Karger AG, Basel

0300-5526/14/0576-0344\$39.50/0 
world [3]. In the following season, the seasonal H1N1 virus continued to circulate at a minimal level but eventually became extinct. However, a return and, in some cases, dominance of seasonal $\mathrm{H} 3 \mathrm{~N} 2$ and influenza $\mathrm{B}$ viruses was observed worldwide $[4,5]$.

In addition to their antigenic evolution, influenza viruses evolve under drug pressure or natural selection, and new virus populations that are resistant to treatment occasionally evolve and sometimes prevail if viral fitness is maintained $[6,7]$. As a result, the use of antiviral drugs like amantadine, an M2-channel inhibitor, has been largely limited due to the surge of amantadine-resistant H3N2 influenza viruses [8]. Neuraminidase inhibitors (NAIs), including oseltamivir and zanamivir, became the drugs of choice for treatment of $\mathrm{H} 3 \mathrm{~N} 2$ influenza infections [9]. The utility of oseltamivir in treating influenza was jeopardized by the worldwide rapid emergence of oseltamivir-resistant seasonal H1N1 viruses during 2007 through 2009 [6, 7]. Nonetheless, this virus was soon replaced by the H1N1p virus, which is largely sensitive to oseltamivir but resistant to amantadine [10]. This has revived the utility of oseltamivir in treating influenza.

Surveillance studies aimed at investigating the evolution and characteristics of influenza viruses worldwide are essential to early detection of new antigenically variant viruses as well as those with decreased antiviral drug susceptibility to allow informed recommendations for vaccination strategies and therapeutic interventions. Influenza surveillance efforts in the Middle East, including Lebanon, the site of this study, have been very limited. Characterization of influenza viruses in this region is critical to compliment the global effort to monitor and control influenza. The H1N1p influenza virus was first detected in Lebanon in May 2009. One study showed that a large outbreak caused by the pandemic virus occurred in October of the same year in Lebanon [11]. The prevalence of H1N1p viruses in the post-pandemic seasons and their susceptibilities to antiviral drugs is unknown in Lebanon.

The goal of this study was to characterize influenza viruses that circulated in Lebanon during two post-pandemic influenza seasons.

\section{Materials and Methods}

\section{Sample Collection}

Nasal swabs were collected from patients presenting with influenza-like illness (ILI) symptoms (i.e. fever of at least $37.5^{\circ}$, cough, rhinorrhea, myalgia, and headache) at a pediatric clinic in Beirut. One swab was immediately tested using a rapid influenza diagnostic test, Quick-Ex Flu kit (Denka Seiken, Japan), and a second swab was stored in virus transport media at $-80^{\circ}$ until further analysis.

Post-Pandemic Influenza in Lebanon

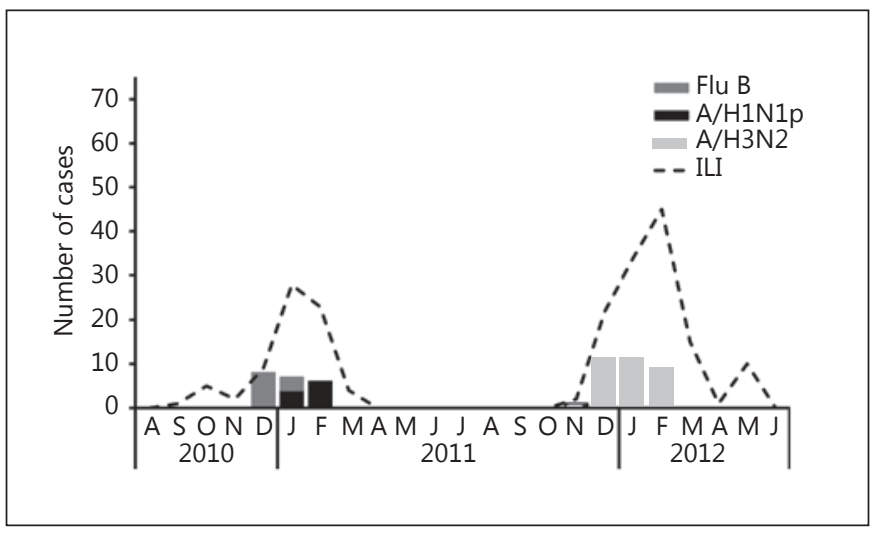

Fig. 1. Graphical representation of influenza cases in Lebanon during the 2010-2012 period. The dotted line represents all ILI cases that were identified during the study period. The letters on the $\mathrm{x}$ axis represent months of the year.

\section{Virus Isolation and Characterization}

$100 \mu \mathrm{l}$ of the swab suspension was inoculated onto Madin-Darby canine kidney (MDCK) cells and incubated at $37^{\circ}$ and $5 \% \mathrm{CO}_{2}$. Influenza virus type and subtype were characterized using cyclingprobe real-time PCR [12-14].

\section{RNA Extraction, PCR, and Sequencing}

Following RNA extraction and reverse transcription $[15,16]$, the HA (HA1 subunit) and neuraminidase (NA) genes were sequenced and aligned using BioEdit software. Phylogenetic trees were constructed using the Neighbor-Joining (MEGA program). Clusters were defined using WHO-designated reference strains [17]. Sequences generated in this study were deposited in the Japanese GenBank database (http://www.ddbj.nig.ac.jp/; accession No. KF000008-62).

\section{Antiviral Drug Susceptibility Testing}

Genotypic analysis of the single-nucleotide polymorphisms M2-S31N and NA-H275Y, which confer resistance to amantadine and oseltamivir, respectively, was performed using cycling probe method $[12,13]$. In vitro antiviral drug susceptibilities of the isolated viruses were determined by measuring the $50 \%$ inhibitory concentrations $\left(\mathrm{IC}_{50}\right.$ ) of oseltamivir (Sequoia Research, UK), zanamivir (Sequoia Research, UK), peramivir (Shionogi, Japan), and laninamivir (Daiichi Sankyo, Japan) using a fluorescencebased NA inhibition assay with methylumbelliferone $\mathrm{N}$-acetylneuraminic acid (MUNANA) as the substrate at a final concentration of $0.025 \mathrm{mM}[18]$.

\section{Results}

A total of 202 samples were collected from patients with ILI during August 2010 through June 2012 (fig. 1). These included 73 samples from the 2010-2011 season and 129 samples from the 2011-2012 season. ILI activity 


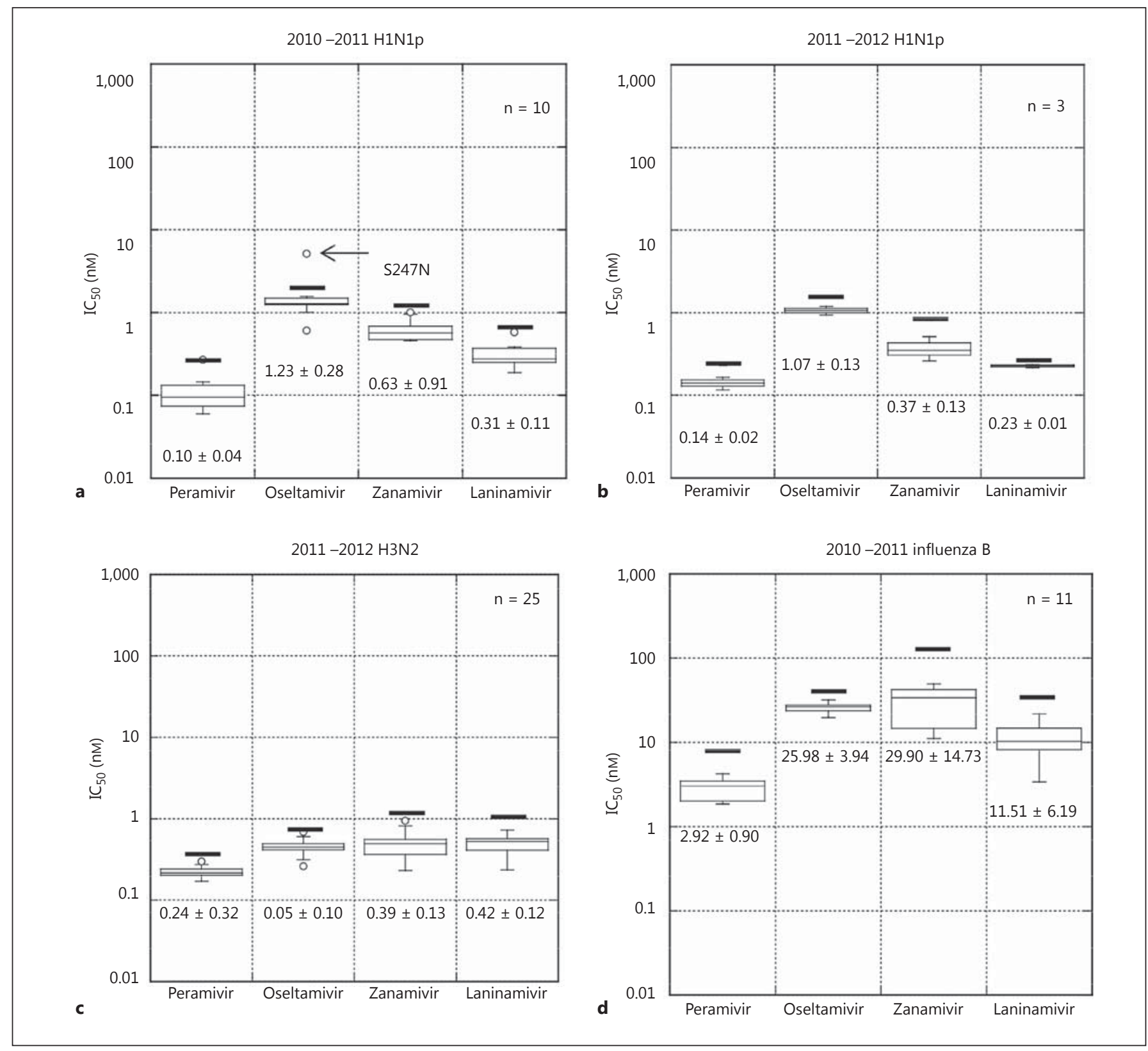

Fig. 2. Box plots of $\mathrm{IC}_{50}$ values of NAIs against 2010-2011 H1N1p (a), 2011-2012 H1N1p (b), 2011-2012 H3N2 (c), and 2010-2011 influenza $\mathrm{B}(\mathbf{d})$ viruses collected in Lebanon. $\mathrm{IC}_{50}$ values of peramivir, oseltamivir, zanamivir, and laninamivir were determined using a fluorescence-based assay with MUNANA as a substrate.

was highest during the months of January and February. In the 2010-2011 season, 12 (17\%) samples tested positive for influenza A and 15 (21\%) samples tested positive for influenza $\mathrm{B}$ using the rapid test kit. Among these, 10 $\mathrm{H} 1 \mathrm{~N} 1 \mathrm{p}$ viruses and 11 influenza B viruses were isolated.
The black bars indicate the 75 th percentile $+3 \times$ interquartile range (3 IQR). Values below the box plot represent mean \pm SD. The mean value was calculated from viruses with $\mathrm{IC}_{50}$ values below the cutoff limit (75th percentile $+3 \mathrm{IQR}$ ). Samples with $\mathrm{IC}_{50}$ greater than the cutoff limit are considered outliers. 
detected. Of these, $23 \mathrm{H} 3 \mathrm{~N} 2$ and $3 \mathrm{H} 1 \mathrm{~N} 1 \mathrm{p}$ viruses were isolated.

Cycling probe real-time PCR analysis revealed that all viruses from both subtypes had the M2-S31N mutation. None of the H1N1p viruses from either the 2010-2011 or 2011-2012 season had the NA-H275Y mutation. Using an in vitro fluorescence-based NA inhibition assay, all $\mathrm{H} 1 \mathrm{~N} 1 \mathrm{p}$ viruses collected in this study were found susceptible to all the tested NAIs with $\mathrm{IC}_{50}$ values of $0.06-0.19$ $\mathrm{nM}$ for peramivir, $0.6-5.11 \mathrm{nM}$ for oseltamivir, $0.46-1.01$ $\mathrm{nM}$ for zanamivir, and $0.19-0.57 \mathrm{nM}$ for laninamivir (fig. 2a, b). One H1N1p isolate (A/Lebanon/10L-21/2011) had a 4 -fold higher oseltamivir $\mathrm{IC}_{50}\left(\mathrm{IC}_{50}=5.11 \mathrm{nM}\right)$ than the average $\mathrm{IC}_{50}(1.18 \mathrm{nM})$ for the rest of the $\mathrm{H} 1 \mathrm{~N} 1 \mathrm{p}$ isolates from both seasons. No difference was observed in $\mathrm{IC}_{50 \text { values }}$ of the other three NAIs against the 10L-21 isolate. For the $\mathrm{H} 3 \mathrm{~N} 2$ viruses, $\mathrm{IC}_{50}$ values were $0.17-0.30 \mathrm{nM}$ for peramivir, $0.94-1.2 \mathrm{nM}$ for oseltamivir, $0.26-0.51 \mathrm{nM}$ for zanamivir, and $0.23-0.73 \mathrm{nM}$ for laninamivir (fig. 2c). Influenza $\mathrm{B}$ isolates had $\mathrm{IC}_{50}$ values that were higher than the influenza $\mathrm{A}$ viruses at $1.85-4.27 \mathrm{nM}$ for peramivir, 19.58-32.05 nM for oseltamivir, $11.10-49.18 \mathrm{nM}$ for zanamivir, and 3.41-21.82 nM for laninamivir (fig. 2d). In summary, the $\mathrm{IC}_{50}$ values suggest that all influenza A viruses isolated in the study are highly susceptible to NAIs. Influenza $\mathrm{B}$ isolates had up to 50 -fold higher $\mathrm{IC}_{50}$ values than influenza A isolates.

Phylogenetic analysis of the HA genes from H1N1p viruses revealed that the 2010-2011 season belonged to five distinct clusters: 1, 5, 6, 7 and 9 (fig. 3a). Cluster 1 included isolates from the 2009 pandemic outbreak with the exception of one isolate (A/Lebanon/10L-21/2011) from the 2010-2011 season, which had 2 additional mutations (S134T and $\mathrm{R} 265 \mathrm{~K}$ ) in the HA gene. Two isolates (A/Lebanon/10L-34/2011 and A/Lebanon/10L-38/2011) from the 2010-2011 season formed a small cluster, designated cluster 9 , characterized by 4 unique mutations (N44D, A267V, G270S, and I272L). None of these mutations belonged to any of the reported antigenic sites. In addition to the 2010-2011 season isolates, cluster 6 also accommodated the 2011-2012 isolates, which accumulated 3 additional HA mutations, including an H144R mutation in the Ca antigenic site and a V158I mutation adjacent to the Sa antigenic site [19]. Overall, the H1N1p viruses analyzed in this study had 6 amino acid mutations in the antigenic sites: 3 in Ca, 2 in Sb, and 1 in Sa, compared with A/California/07/2009. Three of these mutations were not detected in the earlier $\mathrm{H} 1 \mathrm{~N} 1 \mathrm{p}$ viruses.

To determine the genetic basis of the reduced susceptibility to oseltamivir of the 10L-21 (A/Lebanon/10L-21/
2011) isolate from the 2010-2011 season and its genetic relatedness to other isolates, we sequenced the NA genes of all H1N1p viruses isolated in this study. In general, the Lebanese H1N1p NA genes had a clustering pattern that corresponded with their counterpart HA clusters and had 5-7 mutations compared with the A/California/07/2009 vaccine strain (fig. 3). The 10L-21 isolate with reduced susceptibility to oseltamivir belonged to cluster 1 in the NA tree similar to its position in the HA tree. This isolate was characterized by 3 mutations: S247N, N385T, and N386K. The N385T and N386K mutations were located away from the enzyme-active site, suggesting no role in the reduced susceptibility to oseltamivir. The S247N mutation interacts with amino acid residues that in turn interact with oseltamivir and could interfere with stability of the inhibitor in the enzyme-active pocket [20]. The 10L-34 and 10L-38 strains formed a small isolated cluster in the NA tree just like in the HA tree and were characterized by 4 unique mutations: Q313E, T383R, I396T, and S442I.

The HA genes of 8 Lebanese H3N2 influenza viruses collected during the 2011-2012 season were sequenced. All the sequenced viruses were A/Perth/16/2009-like and belonged to the $\mathrm{Vic} / 208$ lineage in the HA phylogeny (fig. 4). Two of the isolates belonged to clade $3 \mathrm{~A}$, and the rest belonged to clade $3 \mathrm{~B}$, which also contained strains from other Middle Eastern countries. These isolates accumulated 9-10 mutations, including 2 antigenic site A mutations (K144N/D and N145S). In case of influenza B, all of the 11 isolates collected during the 2010-2011 season were sequenced. All of these isolates were Victorialike and belonged to the Brisbane/60 clade (fig. 5). The Lebanese strains had 1-2 mutations compared with the B/Brisbane/60/2008 vaccine strain (2009-2012).

\section{Discussion}

In this study, we found that in the first post-pandemic season (2010-2011), both influenza B and A/H1N1p cocirculated at a similar rate. In the 2011-2012 season, the $\mathrm{A} / \mathrm{H} 3 \mathrm{~N} 2$ virus dominated and the $\mathrm{H} 1 \mathrm{~N} 1 \mathrm{p}$ virus co-circulated with a lower prevalence. Similar circulation patterns have been reported elsewhere in the world $[4,21]$. The extinction of seasonal $\mathrm{H} 1 \mathrm{~N} 1$ viruses has been suggested to be a consequence of the original antigenic sin, whereby infection with the H1N1p virus boosted the HA stalk antibodies against the seasonal $\mathrm{H} 1 \mathrm{~N} 1$ virus [22]. A similar phenomenon has been observed when $\mathrm{H} 2 \mathrm{~N} 2$ pandemic viruses, which emerged in 1957, replaced previously circulating $\mathrm{H} 1 \mathrm{~N} 1$ viruses. The $\mathrm{H} 2 \mathrm{~N} 2$ virus circulated for 11 


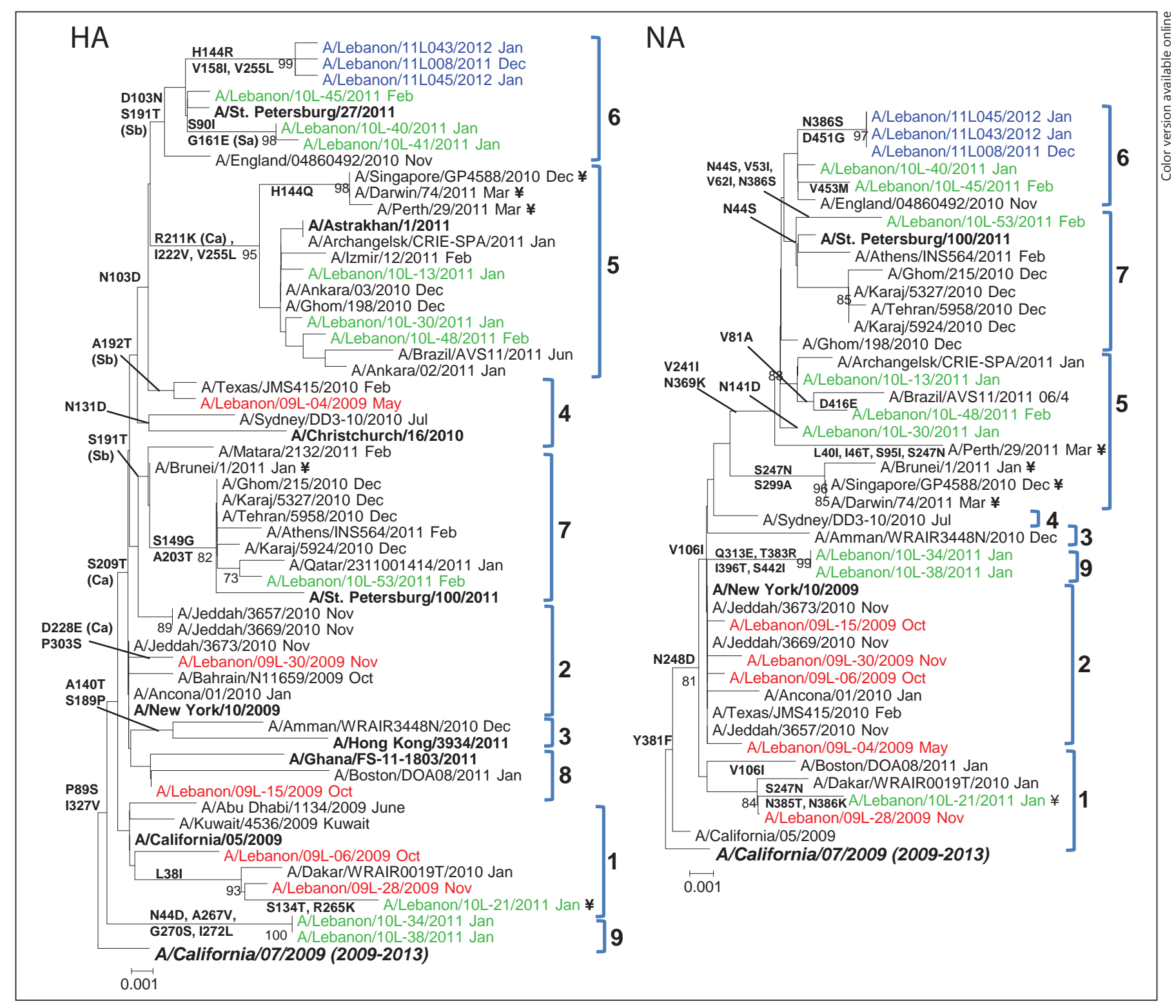

Fig. 3. Phylogenetic analysis of the hemagglutinin (HA1) and NA genes of influenza $\mathrm{A} / \mathrm{H} 1 \mathrm{~N} 1$ pandemic 2009 viruses isolated in Lebanon. The trees were constructed based on the nucleotide sequences using the Neighbor-Joining method with bootstrap analysis of 1,000 replicates. A/California/07/09 was used as the root for the tree and bootstrap values greater than $70 \%$ are shown. Amino acid mutations characteristic of the main clusters or the Lebanese isolates are indicated on the branches. The HA protein antigenic site to which a mutation belongs is in parentheses. Vaccine strains are in bold italics and reference strains are in bold. Lebanese isolates from 2009 (previously reported by Zaraket et al. [11]), 2010-2011, and 2011-2012 seasons are shown in red, green, and blue, respectively. ${ }^{¥}$ Viruses with the S247N mutation. In the NA tree, only reference samples for which the NA gene sequence was available in the GenBank database are included. years until it was replaced by $\mathrm{H} 3 \mathrm{~N} 2$ virus in 1968 due to antibodies specific to the retained N2 subtype [23].

The H1N1p viruses continued to evolve, accumulating mutations in antigenic sites, but remained antigenically similar to the A/California/07/2009 vaccine strain.
In the post-pandemic 2010-2011 season, H1N1p viruses collected in Lebanon were diverse, belonging to multiple clusters, suggesting multiple introductions of this virus into Lebanon. This was in contrast to mostly homologous H1N1p viruses that we detected in previous 
Fig. 4. Phylogenetic analysis of the hemagglutinin (HA1) genes of influenza A/H3N2 viruses isolated in Lebanon. The trees were constructed based on the nucleotide sequences using the Neighbor-Joining method with bootstrap analysis of 1,000 replicates. A/Brisbane/10/2007 was used as the root for the tree and bootstrap values greater than $70 \%$ are shown. Amino acid mutations characteristic of the main clusters or the Lebanese isolates are indicated on the branches. The HA antigenic site to which a mutation belongs is in parentheses. Vaccine strains are in bold italics and reference strains are in bold. Lebanese isolates from 2008-2009 and 2011-2012 seasons are shown in red and blue, respectively.

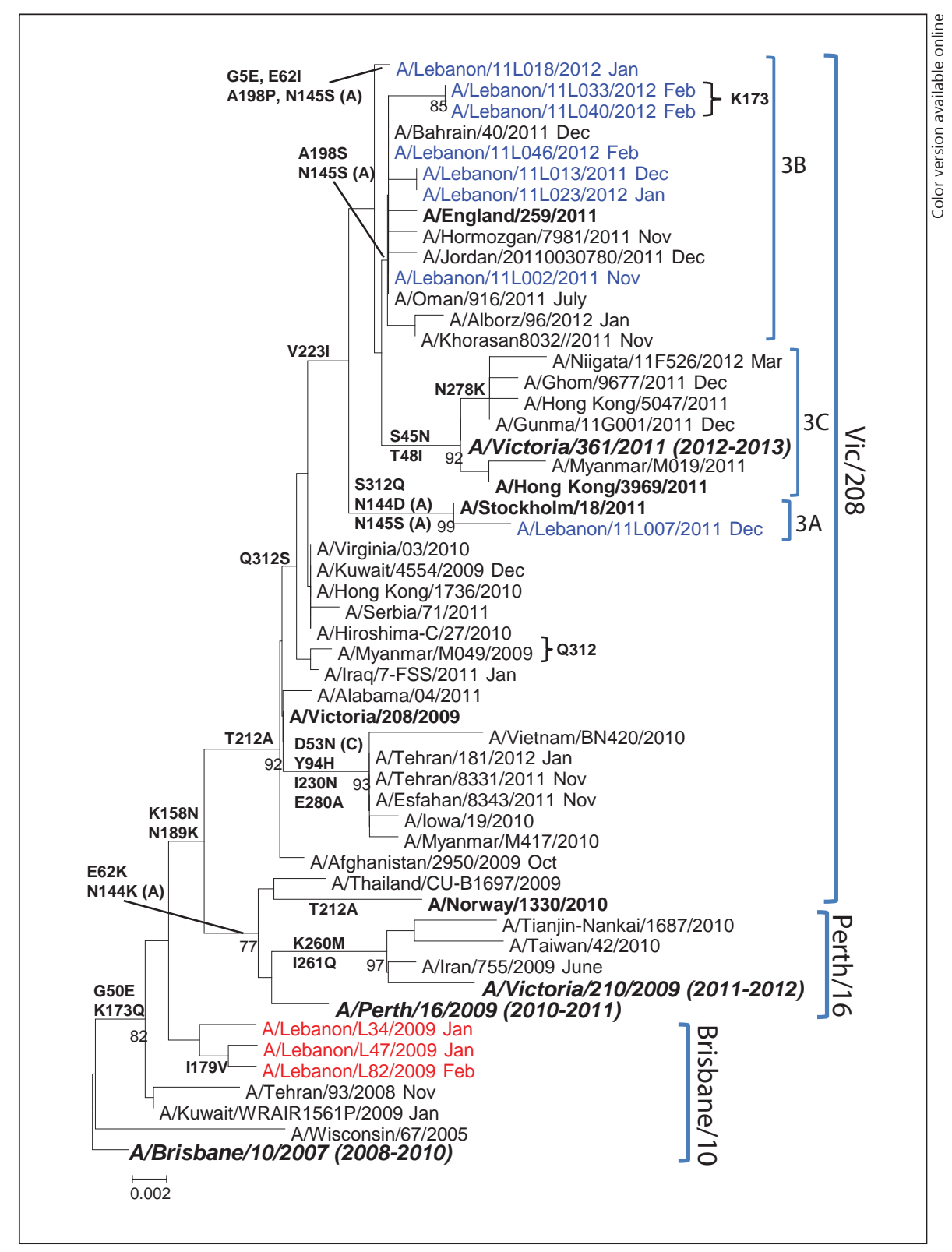

work during the pandemic [11]. In the case of H3N2, all viruses from the 2011-2012 season sequenced in this study belonged to the A/Victoria/208/2011-like lineage, and most of them fell into clade 3B. The Lebanese isolates closely resembled WHO-designated reference strains A/Stockholm/18/2011 and A/England/259/2011 which exhibited 64- and 32-fold reduction, respectively, in their HA inhibition (HAI) titers of sera raised against the A/Victoria/210/2009 vaccine strain [17]. These viruses accumulated several mutations, including 3-4 in the antigenic sites compared with the vaccine strain which explains the antigenic drift marked reduction in their HAI titers.

The prevalence of oseltamivir-resistant $\mathrm{H} 1 \mathrm{~N} 1 \mathrm{p}$ viruses remained low worldwide in the post-pandemic period, albeit at a slight increase from their level during the pandemic period [24-26]. Nonetheless, the rate of oseltamivir resistance is much higher among high-risk groups such as immunocompromised patients, and clusters of high community transmission rates have been reported $[25,27]$. Hurt et al. [28] reported a significant increase in the frequency $\mathrm{H} 275 \mathrm{Y}$ oseltamivir-resistant $\mathrm{H} 1 \mathrm{~N} 1 \mathrm{p}$ virus- 
Fig. 5. Phylogenetic analysis of the hemagglutinin (HA1) genes of influenza B viruses isolated in Lebanon. The trees were constructed based on the nucleotide sequences using the Neighbor-Joining method with bootstrap analysis of 1,000 replicates. The trees are rooted in the middle and bootstrap values greater than $70 \%$ are shown. Amino acid mutations characteristic of the main clusters or the Lebanese isolates are indicated. Vaccine strains are in bold italics and reference strains are in bold. Lebanese isolates from 2008-2009 and 2010-2011 seasons are shown in red and green, respectively.

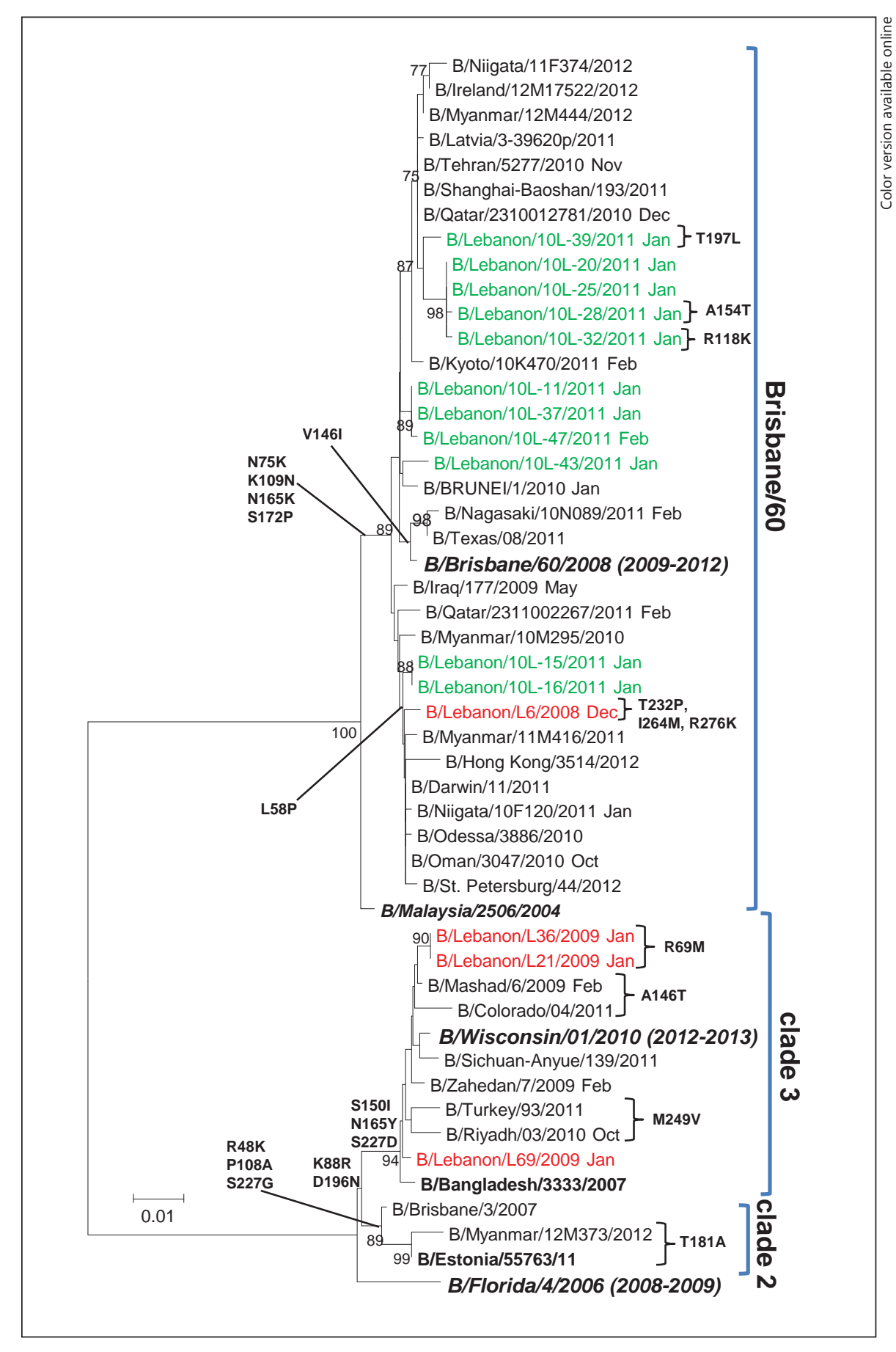

es in a community in Australia. These resistant viruses had three additional NA mutations, V241I, N369K, and N386S, that were suggested to compensate for the destabilizing effect of the H275Y mutation and could, therefore, be permissive for fixing the H275Y mutation in the population. All of the 2011-2012 season H1N1p viruses isolated in Lebanon, despite lacking the $\mathrm{H} 275 \mathrm{Y}$ mutation, had these potentially permissive mutations, and $55 \%$ of the 2010-2011 isolates had 2 of these mutations (V241I and $\mathrm{N} 369 \mathrm{~K})$. Collectively, these data suggest that H1N1p viruses have the capacity to accommodate the H275Y with potentially minimal burden on their fitness. 
We also detected one H1N1p isolate with a 4-fold reduced oseltamivir $\mathrm{IC}_{50}$, due to an NA-S247N mutation, in a patient prior to prescription of oseltamivir. This mutation was reported in 10 and $30 \%$ of H1N1p viruses isolated in Australia and Singapore, respectively, during the same period [20]. The Lebanese S247N isolate was phylogenetically distinct from those isolated in Australia and Singapore. Of note, this mutation was also reported in $\mathrm{H} 5 \mathrm{~N} 1$ viruses isolated in Laos, reducing their susceptibility to oseltamivir by 24 -fold [29]. These reports suggest that the S247N mutation could be naturally selected without antiviral drug pressure and is transmissible. While the $\mathrm{IC}_{50}$ values of $\mathrm{S} 247 \mathrm{~N}$ viruses are well below the biological drug concentrations of oseltamivir [20], the possibility that this mutation gets selected in viruses with the $\mathrm{H} 275 \mathrm{Y}$ mutation is of concern. An isolate with dual NA-S247N and $-\mathrm{H} 275 \mathrm{Y}$ mutations has been already reported and was shown to result in a 6,000-fold increase in oseltamivir $\mathrm{IC}_{50}[20]$, rendering treatment with oseltamivir ineffective.

In conclusion, given the diversity of influenza viruses and the potential for rapid emergence of antiviral drugresistant strains, it is essential to closely monitor influenza viruses both by phenotypic and genotypic assays to quickly identify such emerging mutations. Since the worldwide distribution of antiviral drug-resistant influenza strains varies, it is important to strengthen influenza surveillance at local and regional levels. Such information is critical for maintaining the effectiveness of antiviral drugs for both prophylaxis and treatment of influenza infections.

\section{Acknowledgements}

We thank Dr. George Araj at the American University Medical Center (AUBMC) for his help with sample collection. The present study was funded by the US-Japan Acute Respiratory Infection Panel (The Ministry of Health, Labor, and Welfare, Japan), the US Department of State Biosecurity Engagement Program (Grant \#BEP22033), the American Lebanese Syrian Associated Charities and JSPS Core-to-Core Program, B. Asia-Africa Science Platforms. Ethical approval was obtained from the American University of Beirut Institutional Review Board (PED.GD.02).

\section{Disclosure Statement}

The authors have no conflicts of interest to disclose.

\section{References}

1 Nicholson KG, Wood JM, Zambon M: Influenza. Lancet 2003;362:1733-1745.

-2 Treanor J: Influenza vaccine - outmaneuvering antigenic shift and drift. N Engl J Med 2004;350:218-220.

3 Novel Swine-Origin Influenza AVIT; Dawood FS, Jain S, Finelli L, Shaw MW, Lindstrom S, Garten RJ, Gubareva LV, Xu X, Bridges CB, Uyeki TM: Emergence of a novel swine-origin influenza A (H1N1) virus in humans. N Engl J Med 2009;360:2605-2615.

4 WHO: WHO (2010 March) weekly epidemiology report. http://www.Who.Int/wer. 2010.

5 WHO: WHO (June 2012) weekly epidemiology report. http://www.Who.Int/wer. 2012.

-6 Hurt AC, Ernest J, Deng YM, Iannello P, Besselaar TG, Birch C, Buchy P, Chittaganpitch M, Chiu SC, Dwyer D, Guigon A, Harrower B, Kei IP, Kok T, Lin C, McPhie K, Mohd A, Olveda R, Panayotou T, Rawlinson W, Scott L, Smith D, D'Souza H, Komadina N, Shaw R, Kelso A, Barr IG: Emergence and spread of oseltamivir-resistant a (H1N1) influenza viruses in Oceania, South East Asia and South Africa. Antiviral Res 2009;83:90-93.
Meijer A, Lackenby A, Hungnes O, Lina B, van-der-Werf S, Schweiger B, Opp M, Paget J, van-de-Kassteele J, Hay A, Zambon M; European Influenza Surveillance Scheme: Oseltamivir-resistant influenza virus a (H1N1), Europe, 2007-08 Season. Emerg Infect Dis 2009; 15:552-560.

8 Hayden FG: Antiviral resistance in influenza viruses - implications for management and pandemic response. N Engl J Med 2006;354: 785-788.

9 Ison MG: Antivirals and resistance: influenza virus. Curr Opin Virol 2011;1:563-573.

10 Hurt AC, Chotpitayasunondh T, Cox NJ, Daniels R, Fry AM, Gubareva LV, Hayden FG, Hui DS, Hungnes O, Lackenby A, Lim W, Meijer A, Penn C, Tashiro M, Uyeki TM, Zambon M; WHO Consultation on Pandemic Influenza A (H1N1) 2009 Virus Resistance to Antivirals: Antiviral resistance during the 2009 influenza A H1N1 pandemic: public health, laboratory, and clinical perspectives. Lancet Infect Dis 2012;12:240-248.

11 Zaraket H, Kondo H, Tabet C, Hanna-Wakim R, Suzuki Y, Dbaibo GS, Saito R, Suzuki H: Genetic diversity and antiviral drug resistance of pandemic H1N1 2009 in Lebanon. J Clin Virol 2011;51:170-174.
Suzuki Y, Saito R, Sato I, Zaraket H, Nishikawa M, Tamura T, Dapat C, Caperig-Dapat I, Baranovich T, Suzuki T, Suzuki H: Identification of oseltamivir resistance among pandemic and seasonal influenza $\mathrm{A}$ (H1N1) viruses by an His275Tyr genotyping assay using the cycling probe method. JClin Microbiol 2011;49: 125-130.

13 Suzuki Y, Saito R, Zaraket H, Dapat C, Caperig-Dapat I, Suzuki H: Rapid and specific detection of amantadine-resistant influenza A viruses with a Ser31Asn mutation by the cycling probe method. J Clin Microbiol 2010; 48:57-63.

14 Dapat IC, Dapat C, Baranovich T, Suzuki Y, Kondo H, Shobugawa Y, Saito R, Suzuki H, Japanese Influenza Collaborative Study Group: Genetic characterization of human influenza viruses in the pandemic (20092010) and post-pandemic (2010-2011) periods in Japan. PLoS One 2012;7:e36455.

15 Dapat C, Saito R, Kyaw Y, Naito M, Hasegawa G, Suzuki Y, Dapat IC, Zaraket H, Cho TM, Li D, Oguma T, Baranovich T, Suzuki H: Epidemiology of human influenza $\mathrm{A}$ and $\mathrm{B}$ viruses in Myanmar from 2005 to 2007. Intervirology 2009;52:310-320. 
16 Hoffmann E, Stech J, Guan Y, Webster RG, Perez DR: Universal primer set for the fulllength amplification of all influenza A viruses. Arch Virol 2001;146:2275-2289.

17 NIMR: February 2012 interim report. http:// www.Nimr.Mrc.Ac.Uk/who-influenza-centre/annual-and-interim-reports/. 2012.

18 Hurt AC, Barr IG, Hartel G, Hampson AW: Susceptibility of human influenza viruses from Australasia and South East Asia to the neuraminidase inhibitors zanamivir and oseltamivir. Antiviral Res 2004;62:37-45.

19 Xu R, Ekiert DC, Krause JC, Hai R, Crowe JE Jr, Wilson IA: Structural basis of preexisting immunity to the $2009 \mathrm{H} 1 \mathrm{~N} 1$ pandemic influenza virus. Science 2010;328:357-360.

-20 Hurt AC, Lee RT, Leang SK, Cui L, Deng YM, Phuah SP, Caldwell N, Freeman K, Komadina N, Smith D, Speers D, Kelso A, Lin RT, Maurer-Stroh S, Barr IG: Increased detection in Australia and Singapore of a novel influenza $\mathrm{A}(\mathrm{H} 1 \mathrm{~N} 1) 2009$ variant with reduced oseltamivir and zanamivir sensitivity due to a S247N neuraminidase mutation. Euro Surveill 2011;16

21 WHO: WHO (2010 August) pandemic H1N1 2009. Update 112. http://www.Who.Int/csr/ disease/swineflu/. 2010.

22 Pica N, Hai R, Krammer F, Wang TT, Maamary J, Eggink D, Tan GS, Krause JC, Moran
T, Stein CR, Banach D, Wrammert J, Belshe RB, Garcia-Sastre A, Palese P: Hemagglutinin stalk antibodies elicited by the 2009 pandemic influenza virus as a mechanism for the extinction of seasonal H1N1 viruses. Proc Natl Acad Sci USA 2012;109:2573-2578.

23 Palese P, Wang TT: Why do influenza virus subtypes die out? A hypothesis. MBio 2011; 2:e00150-e00111.

24 Storms AD, Gubareva LV, Su S, Wheeling JT, Okomo-Adhiambo M, Pan CY, Reisdorf E, St George K, Myers R, Wotton JT, Robinson S, Leader B, Thompson M, Shannon M, Klimov A, Fry AM; US Antiviral Resistance Surveillance Working Group: Oseltamivir-resistant pandemic (H1N1) 2009 virus infections, united states, 2010-11. Emerg Infect Dis 2012;18: 308-311.

25 Hurt AC, Hardie K, Wilson NJ, Deng YM, Osbourn M, Gehrig N, Kelso A: Community transmission of oseltamivir-resistant A(H1N1)pdm09 influenza. N Engl J Med 2011;365:2541-2542.

26 Leang SK, Deng YM, Shaw R, Caldwell N, Iannello P, Komadina N, Buchy P, Chittaganpitch M, Dwyer DE, Fagan P, Gourinat AC, Hammill F, Horwood PF, Huang QS, Ip PK, Jennings L, Kesson A, Kok T, Kool JL, Levy A, Lin C, Lindsay K, Osman O, Papadakis G, Rahnamal F, Rawlinson W, Redden C, Ridg- way J, Sam IC, Svobodova S, Tandoc A, Wickramasinghe G, Williamson J, Wilson N, Yusof MA, Kelso A, Barr IG, Hurt AC: Influenza antiviral resistance in the Asia-Pacific region during 2011. Antiviral Res 2013;97:206-210.

- 27 Iioka F, Sada R, Maesako Y, Nakamura F, Ohno H: Outbreak of pandemic 2009 influenza $\mathrm{A} / \mathrm{H} 1 \mathrm{~N} 1$ infection in the hematology ward: fatal clinical outcome of hematopoietic stem cell transplant recipients and emergence of the H275Y neuraminidase mutation. Int J Hematol 2012;96:364-369.

28 Hurt AC, Hardie K, Wilson NJ, Deng YM, Osbourn M, Leang SK, Lee RT, Iannello P, Gehrig N, Shaw R, Wark P, Caldwell N, Givney RC, Xue L, Maurer-Stroh S, Dwyer DE, Wang B, Smith DW, Levy A, Booy R, Dixit R, Merritt T, Kelso A, Dalton C, Durrheim D, Barr IG: Characteristics of a widespread community cluster of H275Y oseltamivir-resistant $\mathrm{A}(\mathrm{H} 1 \mathrm{~N} 1)$ pdm09 influenza in Australia. J Infect Dis 2012;206:148-157.

-29 Boltz DA, Douangngeun B, Phommachanh P, Sinthasak S, Mondry R, Obert C, Seiler P, Keating R, Suzuki Y, Hiramatsu H, Govorkova EA, Webster RG: Emergence of H5N1 avian influenza viruses with reduced sensitivity to neuraminidase inhibitors and novel reassortants in Lao People's Democratic Republic. J Gen Virol 2010;91:949-959. 